\title{
The Contribution of Conductive Network Conversion in Thermal Conductivity Enhancement of Polymer Composite: A Theoretical and Experimental Study
}

\author{
Jingyao Sun, ${ }^{1,2^{*}}$ Xiaowen Zhang, ${ }^{1}$ Qingyuan Du, ${ }^{1}$ Vignesh Murugadoss, ${ }^{4,5}$ Daming $\mathrm{Wu}^{1,3}$ and Zhanhu Guo ${ }^{4 *}$
}

\begin{abstract}
In view of thermal conductive network (TCN) conversion from high thermal dissipation to low thermal dissipation, a new theory of thermal conductivity (TC) enhancement was proposed in this paper. The TC enhancement effect was greatly limited due to the formation of an uncompacted self-assembly network in the composites prepared by traditional compounding methods. In order to achieve the goal of TCN conversion from high thermal dissipation to low thermal dissipation, we combined the TCN densification through space confining forced network assembly (SCFNA) with adding rigid particles. A composite system of polydimethylsiloxane/short carbon fiber/glass bubble (PDMS/SCF/GB) was selected as the modelling object and used to perform verification experiments. On the basis of Nan model, the TCN conversion mathematical model of TC prediction was established. The effects of volume fraction, thermal boundary resistance, thermal contact resistance, aspect ratio and distribution state of fillers on the effective thermal conductivity (ETC) of this PDMS composite were all systematically studied and discussed. In order to verify the accuracy of the model established to predict the TC of PDMS/SCF/GB composite during TCN conversion, a series of experiments were carried out to compare with the model results.
\end{abstract}

Keywords: Thermal conductivity enhancement; Conductive network conversion; Mathematical model; Spatial confining forced network assembled; Rigid particles.

Received date: 25 November 2020; Accepted date: 25 January 2021.

Article type: Research article.

\section{Introduction}

The requirements for thermal conductivity (TC) of material in many frontier application fields are becoming higher and higher with the continuous upgrading of industrial demands. ${ }^{[1-5]}$ Metal has excellent heat conduction ability as a widely used engineering material. However, the application

\footnotetext{
I College of Mechanical and Electrical Engineering, Beijing University of Chemical Technology, Beijing 100029, China.

2 State Key Laboratory of Molecular Engineering of Polymers, Fudan University, Shanghai 200433, China.

${ }^{3}$ State Key Laboratory of Organic-Inorganic Composites, Beijing University of Chemical Technology, Beijing 100029, China.

4 Integrated Composites Laboratory (ICL), Department of Chemical \& Biomolecular Engineering, University of Tennessee, Knoxville, TN 37996, USA.

5 Advanced Materials Division, Engineered Multifunctional Composites (EMC) Nanotech. LLC, Knoxville, TN 37934 USA.

*E-mail: sunjingyao@mail.buct.edu.cn (J. Sun); zguo10@utk.edu (Z. Guo)
}

scope of common metal materials (e.g. Iron and Copper) is greatly limited by their poor flexibility, non-corrosion resisting, and

other defects. Under this situation, polymer composites are treated as one of the most important alternate materials of common metals due to their advantages of low cost, chemical stability, light weight, and ease of processability. ${ }^{[6-8]}$ The thermal conductive polymer composite has already been widely used in many cutting-edge research fields including energy system, ${ }^{[9]}$ Li-ion batteries, ${ }^{[10]}$ electronics, ${ }^{[11-13]}$ photovoltaic (PV) cells, ${ }^{[14]}$ and manufacturing industry. ${ }^{[15]}$

As far as we know, the utilization of thermal conductive fillers (no matter adding single filler or hybrid fillers) could enhance the TCs of polymer composites. ${ }^{[16-18]}$ The commonly used thermal conductive fillers can be roughly divided into three categories: metal fillers (including copper, ${ }^{[19]}$ aluminium, ${ }^{[20]}$ silver ${ }^{[21]}$ iron, ${ }^{[22]}$ etc.), ceramic fillers (including $\mathrm{Al}_{2} \mathrm{O}_{3},{ }^{[23]} \mathrm{AlN},{ }^{[24]} \mathrm{SiC}^{,{ }^{[25]}} \mathrm{BN}$ and $\mathrm{BN}$ nanotube, ${ }^{[26,27]}$ etc.), and carbon series fillers (including graphene, ${ }^{[28]}$ graphene oxide, ${ }^{[29]}$ carbon nanotubes, ${ }^{[30,31]}$ 
graphite, ${ }^{[32]}$ carbon fibers, ${ }^{[33,34]}$ etc.). Previous researches have already paid a lot of attention on the influences of size, shape, filling content, and surface modification of single or hybrid thermal conductive fillers on TCs of polymer composites. ${ }^{[35-}$ 37] The effective promotion of polymer matrix with higher TC on the property enhancement of polymer composite has also been systemically investigated. ${ }^{[38]}$ However, high filling contents would lead to significant decrease of processability and mechanical property of polymer composites, different processing methods (e.g. injection molding, extrusion, etc.) also have their own upper limits of filler contents. ${ }^{[39,40]}$

A lot of efforts have also been done to form continuous thermal conductive network (TCN) in polymer composite under the guidance of thermal conductive path theory. ${ }^{[41]}$ After the formation of continuous TCNs, the TC of polymer composite will increase sharply attribute to more heat flowing along these thermal conductive pathways. There exist three main approaches to form continuous TCN in polymer matrix: (1) rise the filling content of thermal conductive filler to higher than the percolation threshold; (2) construct TCN via selected distribution of thermal conductive fillers in co-continuous two-phase structure; and (3) build segregated structures within polymer composites. Furthermore, different kinds of novel processing approaches (such as sol-gel method, ice-templating method, etc.) have also been developed to construct continuous TCN in polymer composite. For example, Weng et al. reported that inorganicorganic polyethylene glycol (PEG)/silica $\left(\mathrm{SiO}_{2}\right)$ nanocomposites with a low TC of $0.41 \mathrm{~W} / \mathrm{mK}$ were prepared by sol-gel method. ${ }^{[42]} \mathrm{Yu}$ et al. prepared a 3D SiC-nanowire network by ice-templating method, while the $3 \mathrm{D} \mathrm{SiC}$ nanowire/epoxy composites were fabricated by vacuumassisted impregnation of epoxy, which had a relatively low TC of $1.67 \mathrm{~W} / \mathrm{mK}^{[43]}$ The TC enhancement of polymer composites after applied aforementioned approaches is often much lower than the predicted amount. Thus, the investigation of new methods for TC enhancement of polymer composite with better applicability and higher processing efficiency is very essential.

In terms of theoretical model studying, researchers around the world have already proposed different kinds of thermal conduction models and theoretical calculation methods to calculate effective thermal conductivity (ETC) of polymer composite. From initial series-parallel model to the following Maxwell model, Agari Model, Bruggeman model, Nan model and so on, new models and theories are still emerging to meet the requirements for $\mathrm{TC}$ prediction of thermal conductive polymer composites in different situations. However, it is almost impossible to find a single model that can be applied to all situations for TC prediction causes there exist too much influencing factors. All the parameters, including TC of thermal conductive filler, the structure of polymer molecular chains, interface thermal resistance between polymer and thermal conductive filler, and the size, shape, filling content, and treatment of fillers, would affect the TC of polymer composite.

In our present work, a new theory for TC enhancement was proposed basing on the conversion from high to low thermal dissipation TCNs. ${ }^{[33,35]}$ An improved TC model for TCN conversion was also proposed and applied to calculate the TC of polymer composite based on the modified effective medium theory and energy conservation theory. We further applied two different techniques to realize this conversion from high thermal dissipation $\mathrm{TCN}$ to low thermal dissipation TCN, one was the spatial confining forced network assembly (SCFNA), while another one was adding rigid particles. Meanwhile, the thermal property of composite samples will change from low to high TC during this process. In order to verify the availability of this theory, highly thermal conductive polydimethylsiloxane (PDMS) composite using glass bubble (GB) and short carbon fiber (SCF) as fillers were designed and prepared. The thermal conductive SCFs were applied for the construction of continuous TCNs within PDMS matrix, while the GBs were specially selected rigid particles with even lower TC $(0.15 \mathrm{~W} / \mathrm{mK})$ than that of PDMS matrix $(0.27 \mathrm{~W} / \mathrm{mK})$. Herein, the influences of volume fraction effect, thermal boundary resistance, thermal contact resistance, aspect ratio and orientation distribution of fillers on ETC of composite were all systematically studied and discussed. Besides, the comparison between experimental and calculation results of the TCs of PDMS/SCF/GB composites under different compression degrees and filler concentrations were also performed in this research.

\section{Theoretical Model}

\subsection{The establishment of TCN conversion model}

A new thermal conduction model was established basing on the conversion from high to low thermal dissipation TCNs for TC prediction in this paper. ${ }^{[44]}$ There already existed several different prediction models, some of them were found to have a wide applicability, while other models can only be applied for some specific situations. ${ }^{[45]}$ There exist two different types of composite models, the theoretical model and the simulation model. ${ }^{[38,46-49]}$ The simulation models are more suitable for the composites with complicated structures, while the theoretical models can represent the thermal conduction mechanisms. The effective medium approximation (EMA) theory, also named as effective medium theory (EMT), is one of the most commonly used theoretical models. ${ }^{[50]}$ Maxwell-Garnett model is the fundamental model of EMA theory. ${ }^{[51]}$ Some other widely used models, including Every model, ${ }^{[52]} \mathrm{H}-\mathrm{J}$ model, ${ }^{[53]}$ Bruggeman model, ${ }^{[54]}$ Nan model, ${ }^{[55]}$ etc., were all developed for different situations on its basis.

No single model can be used to describe all composite materials because of the complex structure and composition of composites. Thus, a common method is to establish an 
equation group on the basis of a conceptual 'parent' model, which could clarify the changes of structure and composition for specific composite material. Compared with other classical theoretical models, Nan model took the volume fraction, thermal boundary resistance, ${ }^{[52,56]}$ aspect ratio and orientation distribution of fillers into account. Thus, in this paper, the ETC of PDMS/SCF/GB composites was calculated according to Nan model adopting the laws of equivalent TC and minimum thermal resistance.

\subsection{Basic assumptions and the unit cell for calculation}

According to our previous work on SCFNA method, the small amount of GBs would trend to align along the conductive network formed by SCFs under the influences of electrostatic force and the liquid bridge effect between different fillers during SCFNA process to form a low thermal dissipation TCN within the polymer matrix. GB was a hollow spherical structure with air inside and glass outside. During the compression process, they can't be broken up, so that their intrinsic thermal conductivity didn't change. Fig. 1a showed the assumed structure of hybrid SCF and GB fillers in the thermal conductive composite system. Two different kinds of unit cells with periodical arranged fillers (Fig. 1b) showed the possible heat flow pathways for ETC calculation of PDMS/SCF/GB composite.

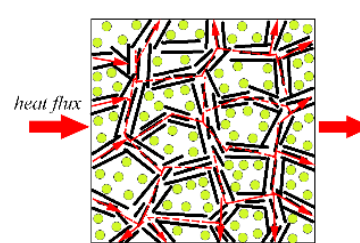

(a)

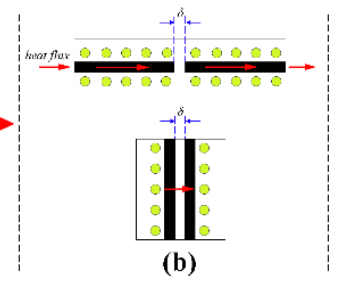

- Carbonfiber $\bigcirc$ Glass bubble $\sqsupset$ Polymer $\quad \delta:$ Polymer layer between carbon fibers

Fig. 1 (a) Assumed structure of hybrid fillers in the polymer composites with low thermal dissipation TCNs; (b) Two kinds of heat flow pathways within polymer composites; (c) The simplified unit cell for calculation.

High thermal dissipation conductive network

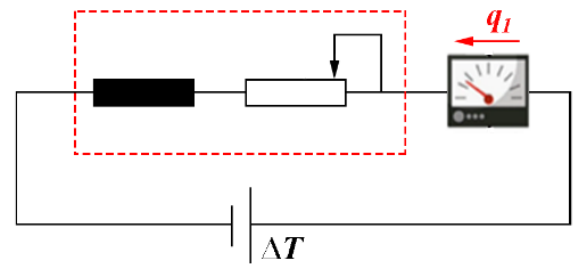

(a)

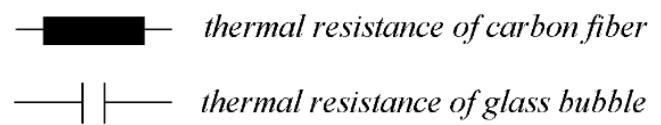

Main assumptions for selecting and simplifying above unit cells are listed below:

(1) The hybrid fillers, including SCF and GB, are evenly distributed in polymer matrix, so the polymer composites can be regarded as a combination of many cuboid unit cells.

(2) For the convenience of analysis and calculation, these two kinds of unit cells can be furtherly simplified into one form, only containing one single SCF and one GB wrapped by polymer matrix (Fig. 1c).

(3) The gap between unit cells will be decreased with the increasing of compression degree during SCFNA process.

(4) GBs are arranged around the TCNs constructed by SCFs. The scattering heat flow will go back to the TCNs during thermal conduction process. Actually, the reduction of thermal resistance of the TCN is just as effective as improving the thermal conductive efficiency of it (Fig. 2).

\subsection{Calculation process of effective thermal conductivity}

The ETC of the PDMS/SCF/GB composite was calculated with three steps. Firstly, calculate the longitudinal TC $\left(k_{z}\right)$ of unit cell. Secondly, transverse TC $\left(k_{x}=k_{y}\right)$ of the unit cell is calculated. Finally, the Nan's equation based on effective medium approach (EMA) is used to calculate the ETC of the PDMS/SCF/GB composite.

As shown in Fig. 3, the TC of one single carbon fiber in three directions is determined by Eq. (1):

$$
\begin{gathered}
k_{x}^{c}=k_{y}^{c}=k_{s} /\left(1+\alpha k_{s} / k_{m}\right) \\
k_{z}^{c}=k_{s}
\end{gathered}
$$

herein, the dimensionless parameter $\alpha$ can be defined as

$$
\alpha=a_{k} / r_{s}
$$

$a_{k}$ in Eq. (2) is Kapitza radius which determined by:

$$
a_{k}=R_{B d} k_{m}
$$

where $k_{s}$ and $k_{m}$ are the TCs of SCF and polymer matrix, and $R_{B d}$ is Kapitza interface thermal resistance across SCF and matrix.

Low thermal dissipation conductive network

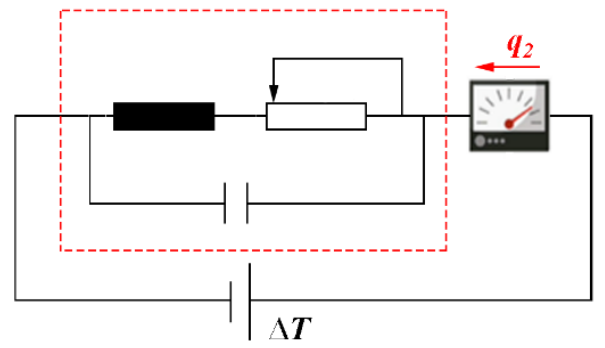

(b)

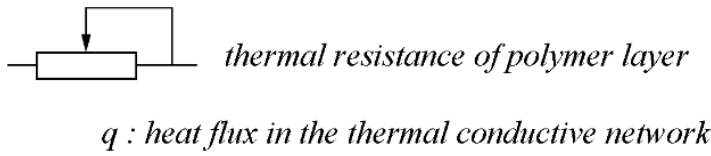

Fig. 2 Schematic diagram of the equivalent thermal resistance in PDMS/SCF/GB composite: (a) high thermal dissipation TCNs; (b) low thermal dissipation TCNs. 


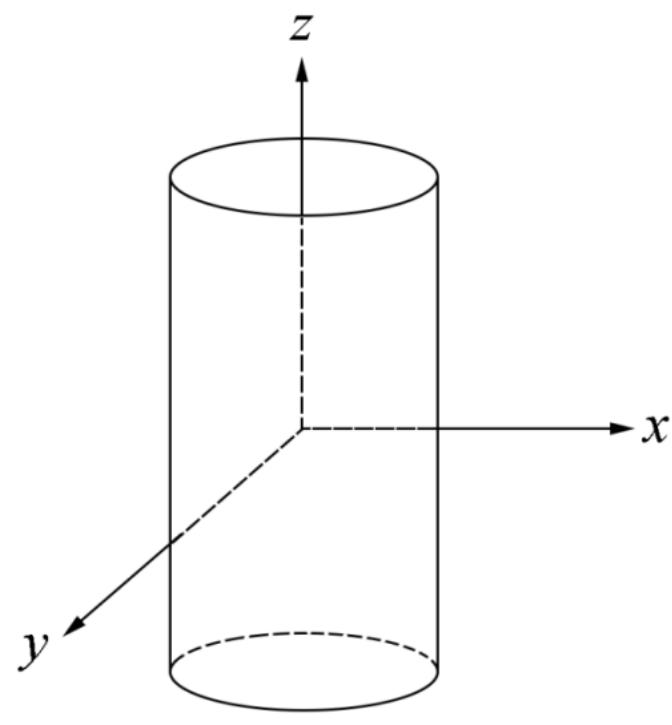

Fig. 3 Dimensions of one single short carbon fiber.

\subsubsection{Longitudinal $\mathrm{TC}$ of the unit cell}

Fig. 4a presents the schematic diagram of heat flux transfer along the longitudinal of continuous SCFs, and the corresponding thermal resistance of single carbon fiber is herein indicated.

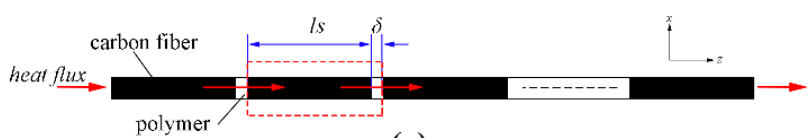

(a)

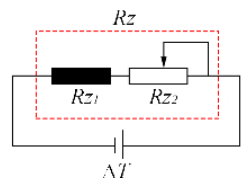

(b)

Fig. 4 (a) Schematic diagram of heat flux transfer along the longitudinal direction; (b) Model of the longitudinal thermal resistance.

Based on the Fourier's law, the thermal resistance can be calculated by,

$$
R=\frac{l}{k \cdot A}
$$

where $k$ is TC of the object, $A$ is heat transfer area perpendicular to the heat flow direction, and $l$ is distance of object along the heat flow direction.

Thus, the longitudinal thermal resistance $R_{z}$ of single carbon fiber can be expressed as

$$
\begin{aligned}
R_{z} & =R_{z 1}+R_{z 2}=\frac{l_{s}}{k_{z}^{c} \cdot A_{z}}+\frac{\delta}{k_{m} \cdot A_{z}} \\
& =\frac{l_{s}}{k_{z}^{c} \cdot \frac{\pi}{4} \cdot d_{s}{ }^{2}}+\frac{\delta}{k_{m} \cdot \frac{\pi}{4} \cdot d_{s}{ }^{2}}
\end{aligned}
$$

where $R_{z 1}$ is longitudinal thermal resistance of SCF, $R_{z 2}$ is thermal resistance of polymer layer between any two SCFs, as shown in Fig. $4 \mathrm{~b}, A_{z}$ is the heat transfer areas of SCFs at cross section that perpendicular to the heat flow direction, $l_{s}$ is the length of SCF, $d_{S}$ is the diameter of SCF, and $\delta$ is the average distance of any two SCFs, as shown in Fig. 4a. Herein, the value of $R_{z 2}$ would change with variation of $\delta$, which made it similar to slide rheostat in traditional circuits.

As shown in Fig. 5a, so as to obtain the equivalent thermal resistance of GBs around single SCF, they are simplified into one glass bubble wrapped in polymer matrix. Fig. $5 b$ indicates the way of thermal conduction in this local system.

The fraction of GBs can be expressed as

$$
\phi_{v, G B}=4 \pi r^{3} / 3 L^{3}
$$

where $\phi_{v, G B}$ is the volume fraction of GBs.

This model can be divided into three parts (as shown in Fig. $5 b)$. Part 1 and part 3 are composed of polymer,

$$
k_{1}=k_{3}=k_{m}
$$

In part 2, the heat flow through polymer matrix $\left(Q_{m}\right)$ and GB $\left(Q_{G}\right)$ in this layer along heat flow direction can be derived as

$$
\begin{aligned}
Q_{G} & =-k_{G} \cdot A_{G} \cdot \frac{d T}{d z} \\
& =-\left(k_{w} \cdot A_{w} \cdot \frac{d T}{d z}+k_{a} \cdot A_{a} \cdot \frac{d T}{d z}\right) \\
Q_{m} & =-k_{m} \cdot A_{m} \cdot \frac{d T}{d z}
\end{aligned}
$$

where $k_{G}$ is the TC of GB, $k_{w}$ is the TC of glass bubble wall, and $k_{a}$ is the TC of air in glass bubble; $A_{m}$ and $A_{G}$ are the heat transfer areas of polymer matrix and GB perpendicular to the heat flow direction at the cross section, respectively; $A_{w}$ and $A_{a}$ are the cross-sectional area of the hollow microsphere wall and the air in GB, respectively;

Let $A=A_{G}+A_{m}=A_{w}+A_{a}+A_{m}$, then $Q$, the overall heat flow can be written as,

$$
\begin{gathered}
Q=Q_{G}+Q_{m}=-\left(k_{w} \cdot A_{w} \cdot \frac{d T}{d z}+k_{a} \cdot A_{a} \cdot \frac{d T}{d z}+k_{m} \cdot A_{m}\right. \\
\left.\cdot \frac{d T}{d z}\right)=-k_{2} \cdot A \cdot \frac{d T}{d z}
\end{gathered}
$$

The following equation can be obtained after integrating Eq. (10) along the heat flow direction.

$$
\begin{aligned}
k_{2} & =\frac{1}{2 r \cdot A}\left(k_{G} \cdot v_{G}+k_{m} \cdot v_{m}\right) \\
& =\frac{1}{2 r \cdot A}\left(k_{w} \cdot v_{w}+k_{a} \cdot v_{a}+k_{m} \cdot v_{m}\right)
\end{aligned}
$$

where $r$ is radius of glass bubble, $\mathrm{k}_{2}$ is the TC of part 2 in Fig. $5 \mathrm{~b}, v_{m}$ is volume of polymer matrix in part 2 , and $v_{w}$ and $v_{a}$ are volumes of hollow microsphere wall and the air in $\mathrm{GB}$, respectively.

Then, the equivalent total thermal resistance can be obtained,

$$
\begin{aligned}
R_{i G} & =R_{1}+R_{2}+R_{3} \\
& =\frac{L-2 r}{k_{m} \cdot A}+\frac{4 r^{2}}{k_{w} \cdot v_{w}+k_{a} \cdot v_{a}+k_{m} \cdot v_{m}}
\end{aligned}
$$

where $\mathrm{R}_{1}, \mathrm{R}_{2}$ and $\mathrm{R}_{3}$ are the thermal resistance of part 1 , part 2 and part 3 in Fig. $5 \mathrm{~b}$, respectively. $\mathrm{R}_{\mathrm{z}}$ and $R_{i G}$ can be calculated by Eqs. (5) and (12). Thus, the equivalent longitudinal thermal resistance of the unit cell $\left(R_{i z}\right)$ was derived as

$$
\frac{1}{R_{i z}}=\frac{1}{R_{z}}+\frac{1}{R_{i G}}
$$


Consequently, the equivalent longitudinal TC of the unit cell $\left(k_{z}\right)$ was obtained using the equation below,

$$
k_{z}=\frac{l_{s}+\delta}{A_{z} \cdot R_{i z}}=\frac{l_{s}+\delta}{\frac{\pi}{4} \cdot d_{s}^{2} \cdot R_{i z}}
$$

Fig. 6 shows the schematic diagram of heat flux transfers along the transverse of continuous SCFs, and the corresponding thermal resistance of single carbon fiber is also indicated.

\subsubsection{Transverse TC of the unit cell}

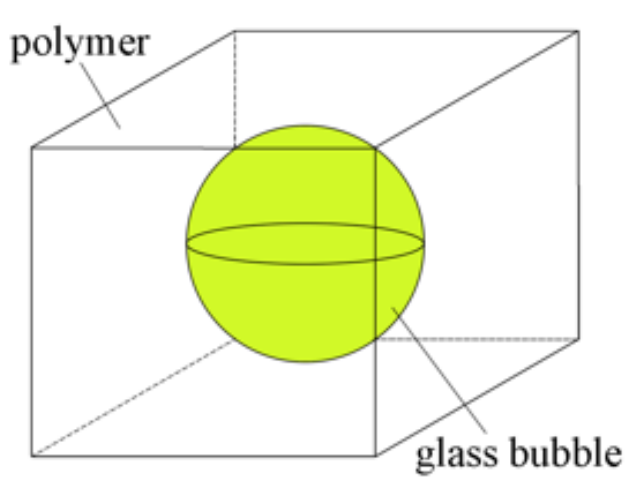

(a)

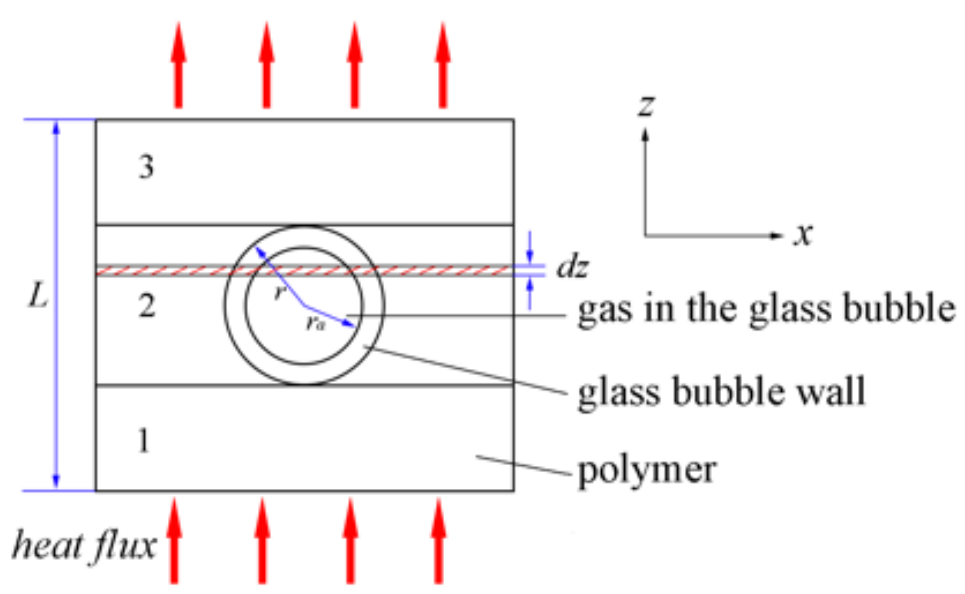

(b)

Fig. 5 Schematic diagram of one glass bubble model: (a) overall geometric pattern of one glass bubble model; (b) sketches for the vertical profile of the glass bubble model.

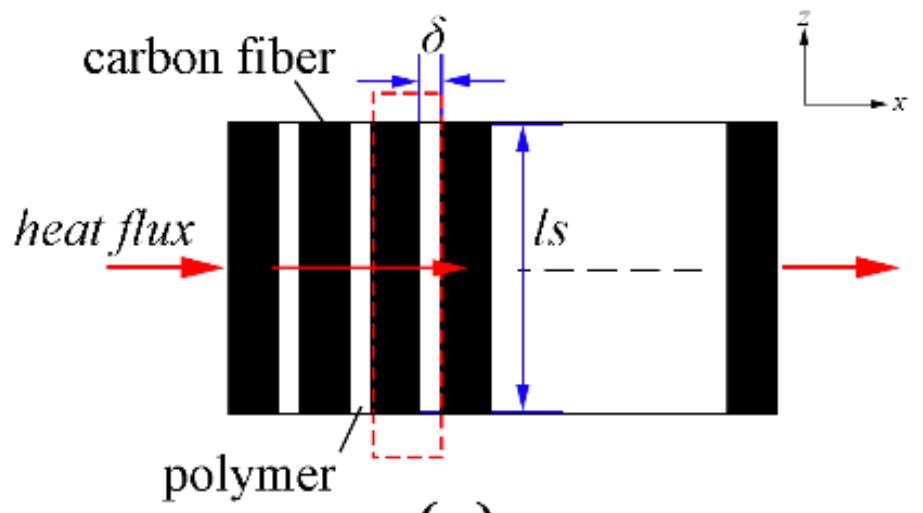

(a)

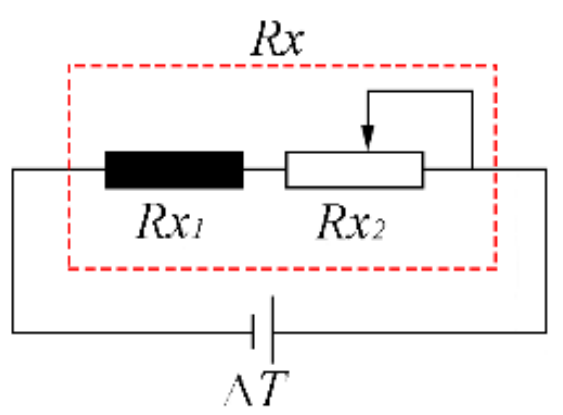

(b)

Fig. 6 (a) Schematic diagram of heat flux conduction along transverse direction; (b) Model of the corresponding thermal resistance.

Similarly, the transverse thermal resistance $R_{i x}$ is calculated first. As shown in Fig. 6b,

$$
R_{x}=R_{x 1}+R_{x 2}
$$

when the heat flux transmits along the transverse direction of $\mathrm{SCF}$, the thermal resistance of polymer matrix between SCFs can be expressed as $R_{x 2}$, which can be calculated using the following equation:

$$
R_{x 2}=\frac{\delta}{k_{m} \cdot A_{x}}=\frac{\delta}{k_{m} \cdot d_{s} \cdot l_{s}}
$$

where $A_{x}$ is the heat transfer area of SCF perpendicular to the heat flow direction at cross section. $R_{x 1}$ is the thermal resistance of the cuboids with SCF inside. As shown in Fig. 7, the heat flow through $\mathrm{SCF} Q_{s}$ and through polymer matrix $Q_{m}^{\prime}$ along the heat flow direction (the $\mathrm{X}$ direction) for a thin layer can be calculated as,

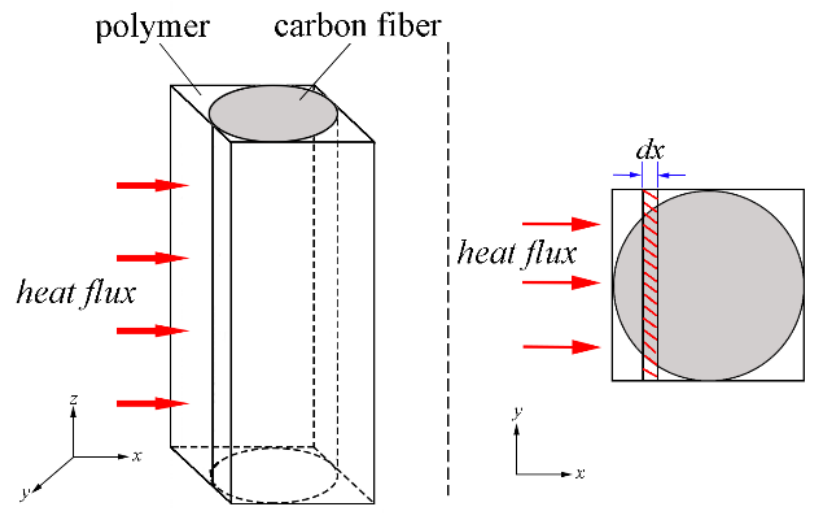

Fig. 7 Heat flux conduction along the transverse direction of short carbon fiber. 


$$
\begin{aligned}
Q_{s} & =-k_{x}^{c} \cdot A_{s} \cdot \frac{d T}{d x} \\
Q_{m}^{\prime} & =-k_{m} \cdot A_{m}^{\prime} \cdot \frac{d T}{d x}
\end{aligned}
$$

where $A_{s}$ and $A_{m}^{\prime}$ are the heat transfer areas of SCF and polymer matrix perpendicular to the heat flow direction at cross section, respectively.

Let $A_{x}=A_{s}+A_{m}^{\prime}$, then $Q^{\prime}$, the overall heat flow, can be expressed as,

$$
\begin{aligned}
Q^{\prime}=Q_{s}+Q_{m}^{\prime} & =-\left(k_{x}^{c} \cdot A_{s} \frac{d T}{d x}+k_{m} \cdot A_{m}^{\prime} \cdot \frac{d T}{d x}\right) \\
& =-k_{x 1} \cdot A_{x} \cdot \frac{d T}{d x}
\end{aligned}
$$

The following equation can be obtained after integrating Eq. (19) along the heat flow direction.

$$
\begin{aligned}
k_{x 1} \cdot A_{x} \cdot d_{s} & =k_{x}^{c} \cdot v_{s}+k_{m} \cdot v_{m}^{\prime} \\
& =k_{x}^{c} \cdot \frac{\pi}{4} d_{s}{ }^{2} l_{s}+k_{m} \cdot\left(d_{s}{ }^{2}-\frac{\pi}{4} d_{s}{ }^{2}\right) l_{s}
\end{aligned}
$$

Thus, $R_{x 1}$ can be calculated as,

$$
R_{x 1}=\frac{d_{s}}{k_{x 1} \cdot A_{x}}=\frac{1}{\left[\frac{\pi}{4} \cdot\left(k_{x}^{c}-k_{m}\right)+k_{m}\right] \cdot l_{s}}
$$

According to Eqs. (15), (16) and (21), $R_{x}$ can be expressed as,

$$
R_{x}=\frac{1}{\left[\frac{\pi}{4} \cdot\left(k_{x}^{c}-k_{m}\right)+k_{m}\right] \cdot l_{s}}+\frac{\delta}{k_{m} \cdot d_{s} \cdot l_{s}}
$$

Considering the influence of GB on the transverse thermal resistance of SCF, the equivalent transverse thermal resistance $R_{i x}$ of the unit cell can be calculated as:

$$
\frac{1}{R_{i x}}=\frac{1}{R_{x}}+\frac{1}{R_{i G}}
$$

Consequently, the equivalent transverse TC of the unit cell $\left(k_{x}\right)$ is obtained.

$$
k_{x}=\frac{d_{s}+\delta}{A_{x} \cdot R_{i x}}=\frac{d_{s}+\delta}{d_{s} \cdot l_{s} \cdot R_{i x}}
$$

\subsubsection{Effective TC of PDMS/SCF/GB composite}

The TCs $k_{x}$ and $k_{z}$ of one single SCF have been modified, and the Nan model is determined by the following equations, $K_{11}^{*}=K_{22}^{*}=$

$$
\begin{gathered}
k_{m} \frac{2+f\left[\beta_{11}\left(1-L_{11}\right)\left(1+\left\langle\cos ^{2} \theta\right\rangle\right)+\beta_{33}\left(1-L_{33}\right)\left(1-\left\langle\cos ^{2} \theta\right\rangle\right)\right]}{2-f\left[\beta_{11} L_{11}\left(1+\left\langle\cos ^{2} \theta\right\rangle\right)+\beta_{33} L_{33}\left(1-\left\langle\cos ^{2} \theta\right\rangle\right)\right]} \\
\beta_{i i}=\frac{k_{i i}-k_{m}}{k_{m}+L_{i i}\left(k_{i i}-k_{m}\right)} \\
\left\langle\cos ^{2} \theta\right\rangle=\frac{\int \rho(\theta) \cos ^{2} \theta \sin \theta d \theta}{\int \rho(\theta) \sin \theta d \theta} \\
L_{11}=L_{22}= \\
\left\{\begin{array}{c}
\frac{p^{2}}{2\left(p^{2}-1\right)}-\frac{p}{2\left(p^{2}-1\right)^{3 / 2}} \operatorname{csch}^{-1} p, \text { for } p>1 \\
\frac{p^{2}}{2\left(p^{2}-1\right)}+\frac{p}{2\left(1-p^{2}\right)^{3 / 2}} \operatorname{csch}^{-1} p, \text { for } p<1 \\
L_{33}=1-2 L_{11} \\
p=l_{s} / d_{s}
\end{array}\right.
\end{gathered}
$$

where 11 and 33 represent the in-plane $(x)$ and through-plane $(z)$ directions, $f$ is the volume fraction of SCF, $p$ is aspect ratio, $k_{i i}$ represents the equivalent $\mathrm{TC}$ along the $i i$ symmetrical axis of the unit cell of polymer composite, i.e. $k_{11}=k_{22}=k_{x}=k_{y}, k_{33}=k_{z}$, and $\theta$ is the angle between the $z$ axis of composite and the symmetrical axis $z$ of local unit cell. ${ }^{[55]}$

\section{Experimental \\ 3.1. Materials}

A SYLGAR ${ }^{\circledR} 184$ PDMS prepolymer kit was purchased from Dow Corning Corp (USA) and applied as polymer matrix using a weight ratio of 1:10 (curing agent to silicone elastomer). The SCFs with $6 \mathrm{~mm}$ length and $11 \mu \mathrm{m}$ diameter were provided by Shanghai Q-Carbon material Co. Ltd., (China), while the GBs with $\sim 20 \mu \mathrm{m}$ diameter were provided by $3 \mathrm{M}$ company (USA). The densities of GBs and SCFs are 0.46 and $2.20 \mathrm{~g} / \mathrm{cm}^{3}$, respectively. The TCs of PDMS, GB and $\mathrm{SCF}$ are $0.27,0.15$, and $550 \mathrm{~W} / \mathrm{mK}$, respectively.

\subsection{The preparation of PDMS/SCF/GB composites}

Schematic diagram of the preparation process of PDMS/SCF/GB composites was presented in Fig. 8. In this work, the PDMS/SCF(30 wt\%)/GB composites with different GB contents $(0,1,2,3,4$ and $8 \mathrm{wt} \%)$ were prepared. Composite samples with different compression ratios (CR) were also prepared. CR was a self-defined parameter to indicate the compression degree of samples during SCFNA process and can be calculated by Eq. (31):

$$
C R=\frac{2 \mathrm{~mm}}{\text { thickness of sample }}=\frac{2}{h}
$$

where $h$ is the thickness composite sample.

In this work, the $2.0 \mathrm{~mm}$ thick $\mathrm{PDMS} / \mathrm{SCF} / \mathrm{GB}$ composites fabricated by the traditional compounding methods were considered as standard samples with high thermal dissipation conductive networks. The whole preparation process of low thermal dissipation PDMS/SCF/GB composites can be divided into several simple steps. First, the PDMS/SCF compound was prepared with the HAAKE inner mixer for $10 \mathrm{~min}$ at $40 \mathrm{rpm}$ rotation speed under room temperature, while an agitator was applied to prepare the PDMS/GB compound for $30 \mathrm{~min}$ at $50 \mathrm{rpm}$ rotation speed for $30 \mathrm{~min}$. Second, air bubbles in the PDMS/SCF and PDMS/GB compounds were both removed in a vacuum environment using a vacuum oven. Third, the two different compounds mentioned above were mixed with the agitator furtherly to obtain homogenously dispersed PDMS/SCF/GB compound. Then, the PDMS/SCF/GB compound was compressed firstly to $2 \mathrm{~mm}$ thick for $60 \mathrm{~s}$ within the compression mold using a self-designed hot embossing equipment. ${ }^{[57-60]}$ Subsequently, the samples were furtherly compressed to different thicknesses of $0.6,0.4,0.2$, and $0.1 \mathrm{~mm}$. The so-called "TCN conversion" will happen due to the joint influences of forced compression and adding GBs. Finally, the PDMS/SCF/GB compound was heated up to $120^{\circ} \mathrm{C}$ for curing within $30 \mathrm{~min}$ and get the final products. 


\subsection{Characterization}

A scanning electronic microscope (Hitachi S-4700, SEM) and a metallographic microscope (XJ-55C, Shanghai Puzhe Photoelectric Instrument Co. Ltd.,) were applied to characterize the morphology of PDMS/SCF/GB composite. The TCs of PDMS/SCF/GB composites were tested under room temperature and calculated by $k=\alpha \cdot C_{p} \cdot \rho$, where $\alpha$ is in-plane TC $\left(\mathrm{mm}^{2} / \mathrm{s}\right)$, measured according to laser flash technology using the Netzsch system (LFA 467, German), $\rho$ is the density of composite sample $\left(\mathrm{g} / \mathrm{cm}^{3}\right)$, and $C_{p}$ is the specific heat $(\mathrm{J} /(\mathrm{g} \cdot \mathrm{K}))$, tested using DSC Pyris 1 (PerkinElmer, America).

\section{Results and discussion}

\subsection{Properties of the PDMS/SCF/GB composites}

As shown in Fig.8, the self-assembled network by free compression was uncompacted networks with the insufficient connections between the fillers and the poor orientation of the thermal conductive network. Afterwards, the forcedassembled network was formed by SCNFA process and the TCs of composite samples improved. The TCs of composite samples prepared with different filling contents and compression ratios were listed in Table 1 . The $2.0 \mathrm{~mm}$ thick composite samples were fabricated using the method of traditional compounding and presented relatively low thermal conductivities (only $1.053 \mathrm{~W} / \mathrm{mK}$ ) even through 30 wt $\%$ SCFs were added to build continuous TCNs in polymer matrix. The reason was that the TCNs in these samples were uncompacted self-assembled networks. The uncompacted TCN was a high dissipation channel for heat flux formed by the simple accretion of polymer matrix and conductive fillers. Continuous heat absorbing by polymer matrix and heat leaking from TCN to surrounding polymer would occur when heat flux transmitted along these high thermal dissipation conductive networks. After excessive amount of GBs (e.g. $8 \mathrm{wt} \%$ ) were added into this composite system with uncompacted high thermal dissipation conductive networks, its thermal conductivity furtherly decreased to $0.252 \mathrm{~W} / \mathrm{mK}$, even slightly lower than the value of pure PDMS $(0.27 \mathrm{~W} / \mathrm{mK})$.

The TC of PDMS/SCF/GB composite increased sharply with increasing CR. Take the PDMS/SCF $(30 \mathrm{wt} \%) / \mathrm{GB}(0$ $\mathrm{wt} \%)$ composite as an example, the TC presented a 4.69 times enhancement to $5.991 \mathrm{~W} / \mathrm{mK}$ while the CR of samples increased from 1 to 3.33 . That is, the thickness of composite samples decreased from $2.0 \mathrm{~mm}$ to $0.6 \mathrm{~mm}$ during SCFNA process and the self-assembled network with a high thermal dissipation started to convert into forced-assembled network with a low thermal dissipation. In cases of higher CRs $(\mathrm{CR}=5,10$, and 20), the same composite system presented even higher thermal conductivities of $8.227,10.706$, and $11.423 \mathrm{~W} / \mathrm{mK}$, which were $6.81,9.17$, and 9.85 times enhancements, respectively. It indicated that the TCNs formed by SCFs had become more and more compact with increasing CRs and the initial high thermal dissipation TCNs had already converted into primary low thermal dissipation TCNs. The matrix within polymer layers between SCFs will be squeezed out from the uncompacted TCNs with increasing the CRs in SCFNA process and the dissipation of heat flux will be reduced.

Besides, the addition of appropriate amount of GBs can furtherly enhance the TC of the PDMS/SCF/GB composite. For example, the TC of aforementioned PDMS/SCF $(30$ $\mathrm{wt} \%) / \mathrm{GB}(0 \quad \mathrm{wt} \%)$ composite presented an $\sim 13 \%$ enhancement from $11.423 \mathrm{~W} / \mathrm{mK}$ to $13.004 \mathrm{~W} / \mathrm{mK}$ after the addition of $2 \mathrm{wt} \% \mathrm{~GB}$ under the condition of $\mathrm{CR}=20$. When only $1 \mathrm{wt} \%$ GBs were added, the TCs of PDMS/SCF 30 $\mathrm{wt} \%) / \mathrm{GB}(1 \mathrm{wt} \%)$ composites were increased by $7.44,9.84$, and 10.90 times to $8.892,11.417,12.532 \mathrm{~W} / \mathrm{mK}$ when the CRs were 5, 10, and 20, respectively. The replacement of partial polymer matrix around the TCNs with a high thermal dissipation by lower thermal dissipation GBs was the main

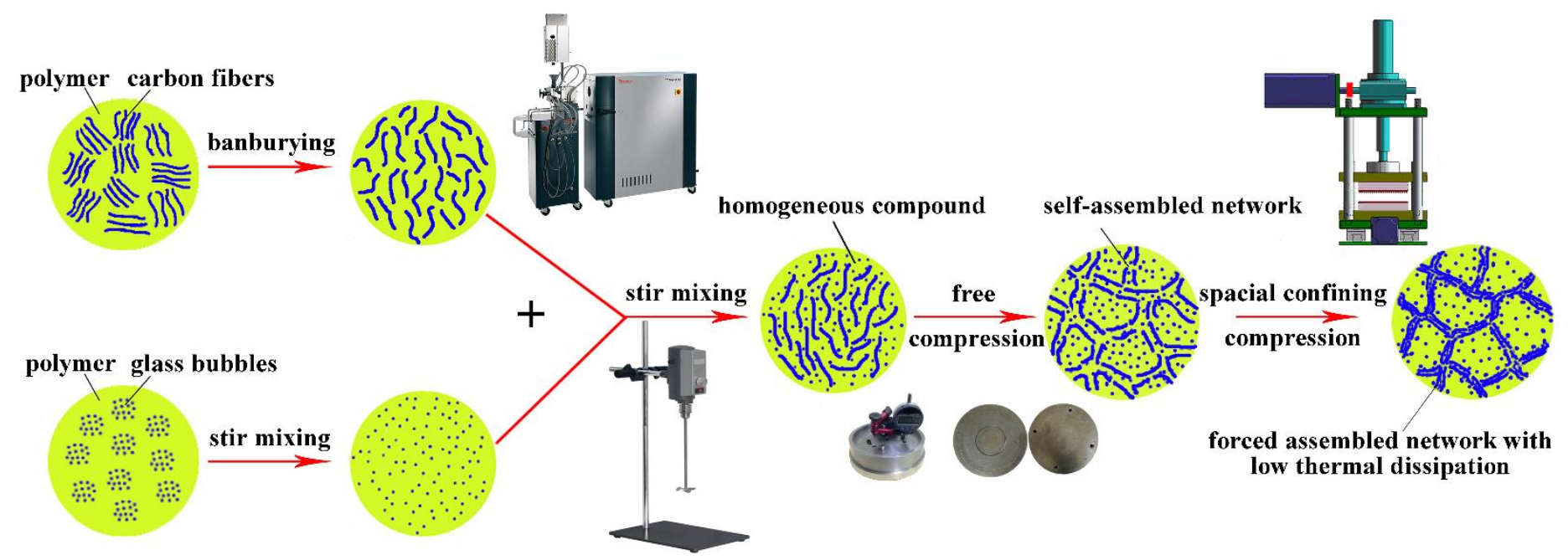

Fig. 8 The schematic diagram of preparation process of low thermal dissipation PDMS/SCF/GB composites and the equipment needed. 
Table 1. TCs of composite samples with different CRs and GB contents.

\begin{tabular}{|c|c|c|c|c|c|}
\hline \multirow[b]{2}{*}{ Methods } & \multicolumn{2}{|c|}{ Filling contents (wt\%) } & \multirow{2}{*}{$\begin{array}{c}\text { Thickness } \\
(\mathbf{m m})\end{array}$} & \multirow{2}{*}{$\begin{array}{c}\text { Compression } \\
\text { Ratio } \\
\end{array}$} & \multirow{2}{*}{$\begin{array}{c}\text { Thermal conductivity } \\
(\mathrm{W} / \mathbf{m K})\end{array}$} \\
\hline & SCF & GB & & & \\
\hline $\begin{array}{c}\text { Traditional } \\
\text { compounding } \\
\text { method }\end{array}$ & 30 & 0 & 2 & ---- & 1.053 \\
\hline \multirow{19}{*}{$\begin{array}{l}\text { Spatial confining } \\
\text { forced network } \\
\text { assembly (SCFNA) }\end{array}$} & \multirow{5}{*}{30} & 0 & \multirow{5}{*}{0.1} & \multirow{5}{*}{20} & 11.423 \\
\hline & & 1 & & & 12.532 \\
\hline & & 3 & & & 11.302 \\
\hline & & 4 & & & 10.050 \\
\hline & & 8 & & & 8.912 \\
\hline & \multirow{2}{*}{30} & 0 & \multirow{2}{*}{0.2} & \multirow{2}{*}{10} & 10.706 \\
\hline & & 1 & & & 11.417 \\
\hline & \multirow{6}{*}{30} & 0 & \multirow{6}{*}{0.4} & \multirow{6}{*}{5} & 8.227 \\
\hline & & 1 & & & 8.892 \\
\hline & & 2 & & & 7.820 \\
\hline & & 3 & & & 7.319 \\
\hline & & 4 & & & 7.528 \\
\hline & & 8 & & & 6.693 \\
\hline & \multirow{4}{*}{30} & 0 & \multirow{4}{*}{0.6} & \multirow{4}{*}{3.33} & 5.991 \\
\hline & & 1 & & & 6.420 \\
\hline & & 2 & & & 6.439 \\
\hline & & 3 & & & 6.881 \\
\hline & \multirow{2}{*}{30} & 4 & \multirow{2}{*}{2} & \multirow{2}{*}{1} & 0.937 \\
\hline & & 8 & & & 0.252 \\
\hline
\end{tabular}

reason for this increasement. Moreover, more heat flux would be scattered back to networks after this replacement by the aid of forced-assembled TCN. The heat flux scattering and volume exclusion effects of appropriate number of GBs in the PDMS/SCF/GB composites can significantly reduce the dissipation and absorption of heat flux by surrounding polymer. However, excessive amount of GBs would lead to large reduction of thermal conductivity because part of the thermal insulation GBs tended to locate inside the TCNs. The TCNs within PDMS/SCF/GB composites would be partially destroyed and the heat transfer process would be blocked. With the effect of the two aspects above (SCFNA process and adding appropriate amount of rigid particles), the high thermal dissipation TCNs within the $\mathrm{PDMS} / \mathrm{SCF} / \mathrm{GB}$ composite were completely convert into low thermal dissipation ones.
The cross-section views of the PDMS-based composite samples with thermal conductive networks were shown in Fig. 9. Herein, the filling content of SCF in PDMS/SCF/GB composites was all set at $30 \mathrm{wt} \%$ as the influence of SCF content on thermal conductivities of polymer composites in SCFNA process had already been systematically investigated in our previous works ${ }^{[33,35]}$. Fig. 9a to 9c showed the morphology evolution of $\mathrm{PDMS} / \mathrm{SCF}(30 \quad \mathrm{wt} \%) / \mathrm{GB}$ composites with different filling contents of GBs. While the GB content raised from 0 (Fig. 9a) to $2 \mathrm{wt} \%$ (Fig. 9b), not much morphological changes had occurred in the thermal conductive networks and the small amount of GBs tended to distribute along the SCF networks. Much more GBs could be found in Fig. 9c after the GB content raised to $8 \mathrm{wt} \%$. Some GBs even located between two neighbouring SCFs, which meant the thermal conductive networks were partially 
destroyed and the thermal conductivity would present large reductions in these situations. Meanwhile, Fig. 9c to 9e presented the morphology evolution of PDMS/SCF(30 $w t \%) / G B(8$ wt $\%)$ composites with different CRs. The distribution of SCFs became much more orderly with the increasing of CRs. Fig. 9f showed the images of $\mathrm{PDMS} / \mathrm{SCF} / \mathrm{GB}$ composite with low thermal dissipation TCNs under normal and fold states.

SCFs and uncured PDMS matrix were applied to perform verified experiments with the help of an optical microscope to observe the low thermal dissipation forced-assembled networks (as shown in Fig. 8). Fig. 10 showed the morphology evolution of SCF networks in uncured PDMS during the SCFNA process. Forced-assembled networks can be observed obviously in Fig. $10 \mathrm{~b}(\mathrm{CR}=20)$, while the aggregation of SCFs was still in its primary stage in Fig. 10a $(\mathrm{CR}=5)$.

\subsection{Comparisons between calculation results and experimental data}

For continuous SCFs, the values of $p, L_{11}, L_{33}$, and $R_{B d}$ in this paper are set as $p \gg 1, L_{11}=0.5, L_{33}=0$, and $R_{B d}=$
$8 \times 10^{-8} \mathrm{~m}^{2} \mathrm{~K} / \mathrm{W}$. The values $\delta$ and $l_{s}$ were determined by SEM images. As reported previously, the orientation characteristic $\left\langle\cos ^{2} \theta\right\rangle$ is equivalent to $0,1 / 3$, and 1 for the composite samples with SCFs completely oriented along the in-plane (X) direction, distributed randomly, and along through-plane $(Z)$ direction. That is, when the value of $\left\langle\cos ^{2} \theta\right\rangle$ is 0 or 1 , the SCFs are completely parallelly or perpendicularly oriented to the direction of heat flux. The TC of PDMS/SCF/GB composite would respectively reach the maximum and minimum in the aforementioned two cases.

Based on the analysis above, the influence of GB was relied on the application of SCFNA method. Therefore, comparisons between calculation results and experimental data are only performed and discussed in the cases of $\mathrm{CR}=20,10$ and 5. Fig. $11 \mathrm{a}$ to $11 \mathrm{c}$ presents the comparisonbetween the calculated and measured $\mathrm{k}$ of PDMS/SCF/GB composite. As the unit of SCF and GB contents in $\mathrm{PDMS} / \mathrm{SCF} / \mathrm{GB}$ composites were both volume fraction (vol\%) in the establishment process of theoretical model, the unit of GB content was also selected as vol $\%$ for the convenience of discussion in Fig. 11. Thus, the filling contents of GBs in $\mathrm{PDMS} / \mathrm{SCF} / \mathrm{GB}$ composites changed
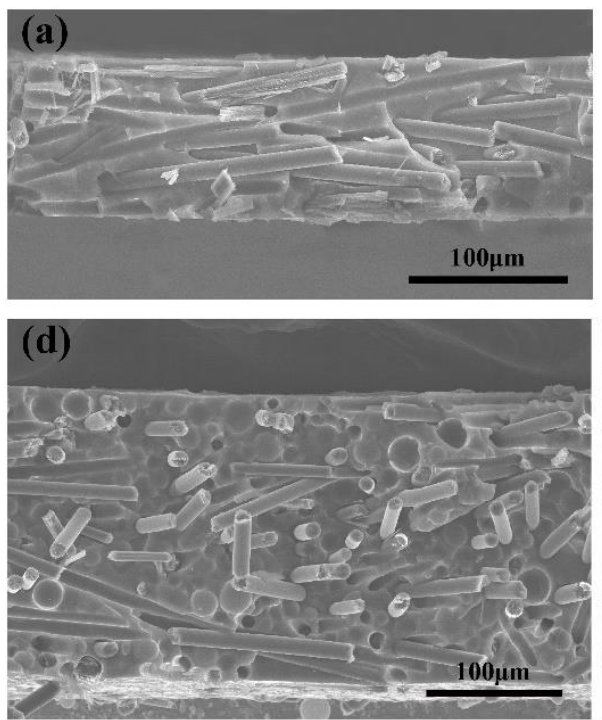

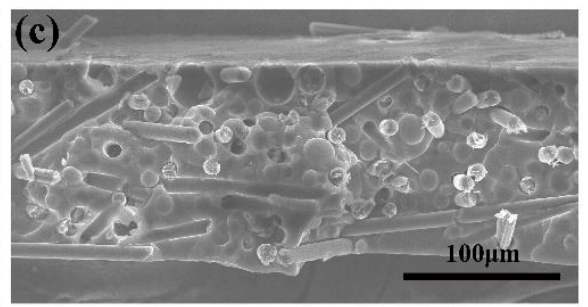

(f)

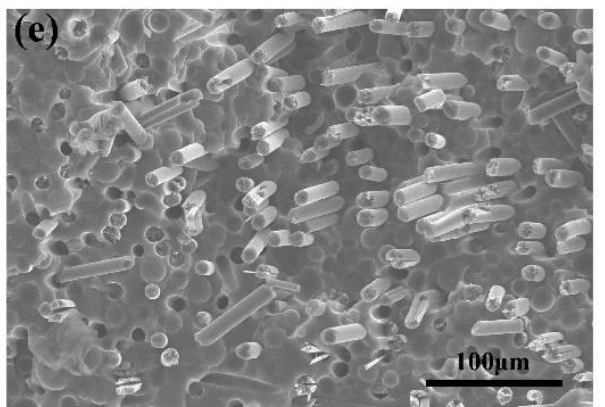

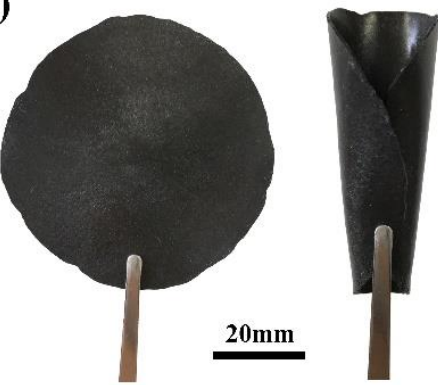

Fig. 9 Through-plane (a-f) morphology evolution of PDMS/SCF/GB composites: (a) $\mathrm{CR}=20$, GB content=0; (b) $\mathrm{CR}=20$, GB content=2; (c) $\mathrm{CR}=20$, GB content=8; (d) $\mathrm{CR}=10$, GB content=8; (e) $\mathrm{CR}=5$, GB content=8; and (f) Photos of PDMS/SCF/GB composite sample under normal (left) and fold (right) states.
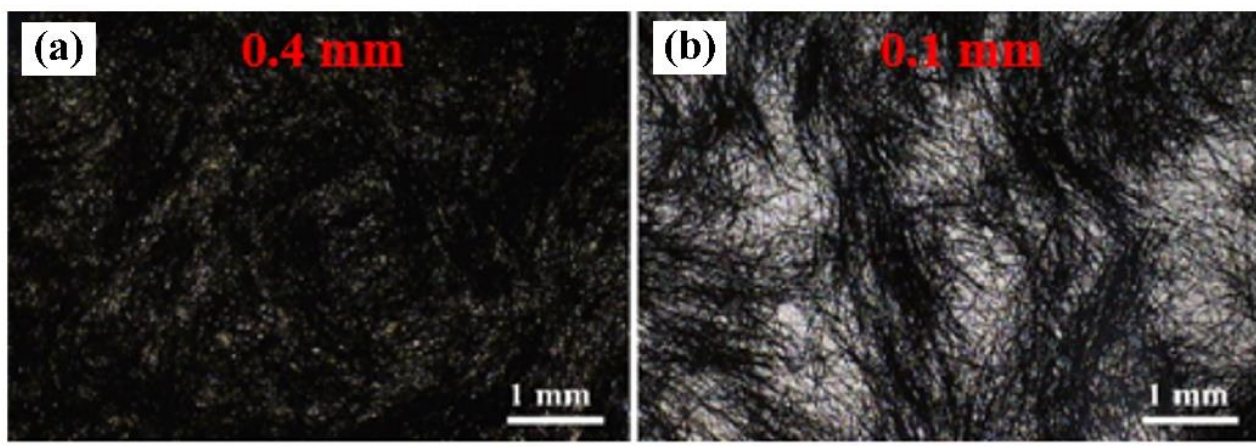

Fig. 10 In-plane morphology evolution of SCF networks in uncured PDMS matrix during SCFNA process: (a) $0.4 \mathrm{~mm}$ thick sample $(\mathrm{CR}=5)$; (b) $0.1 \mathrm{~mm}$ thick sample $(\mathrm{CR}=20)$. 


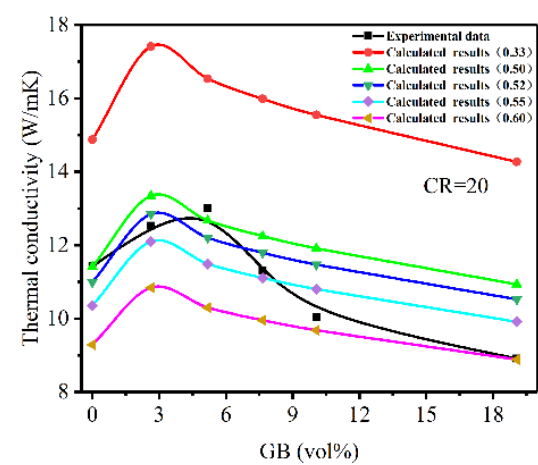

(a)

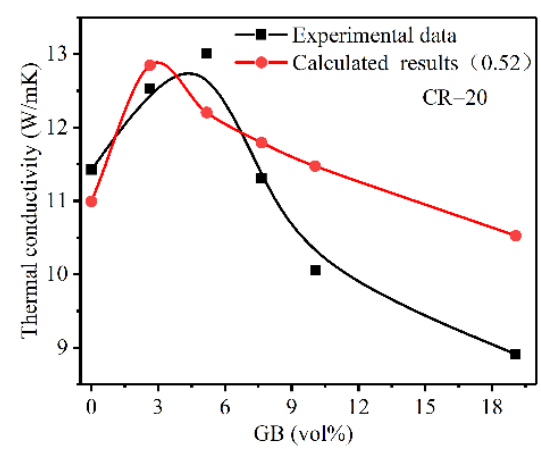

(d)

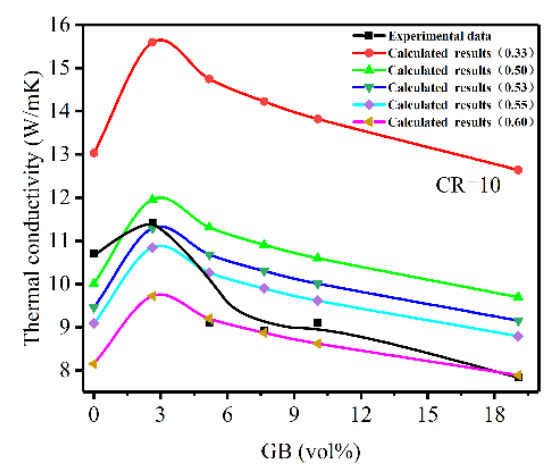

(b)

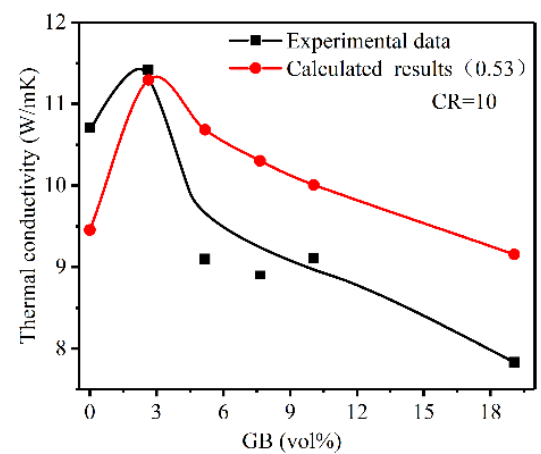

(e)

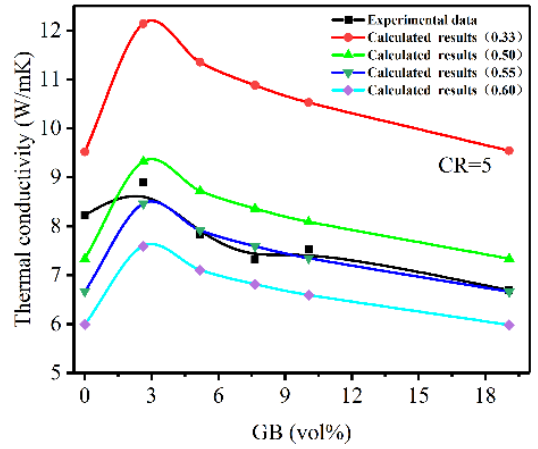

(c)

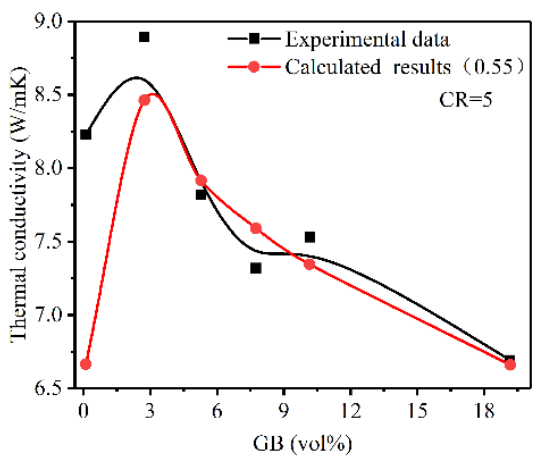

(f)

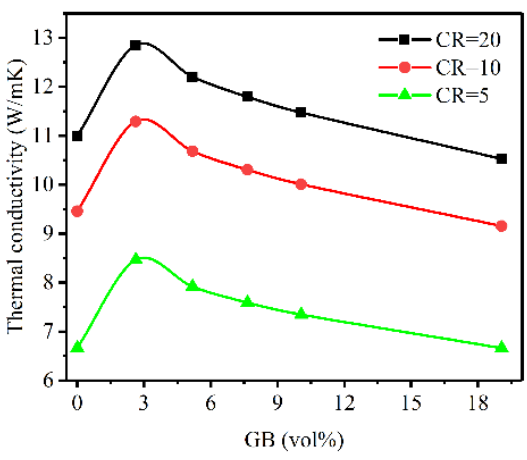

(g)

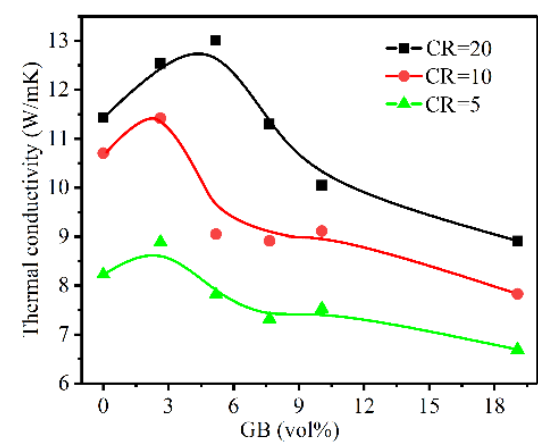

(h)

Fig. 11 Comparison of calculated results of the proposed TC model with different $\left\langle\cos ^{2} \theta\right\rangle$ and CRs: (a) $\mathrm{CR}=20$; (b) $\mathrm{CR}=10$; (c) $\mathrm{CR}=5$. Comparison of calculated results and experimental data under different CRs: (d) $\mathrm{CR}=20$; (e) $\mathrm{CR}=10$; (f) $\mathrm{CR}=5$. (g) Comparison of calculation results under three different CRs; (h) Comparison of experimental data under three different CRs.

from $1,2,3,4$, and 8 wt $\%$ into $2.63,5.18,7.65,10.06$, and 19.06 vol\%. As shown in Fig. 11, the experiment result of TC is much lower than calculation data when the orientation characteristic $\left\langle\cos ^{2} \theta\right\rangle$ is equivalent to $1 / 3$. The reason is that when SCFs are completely randomly dispersed in the polymer matrix, it is much easier for them to contact with each other and form TCNs because of their high aspect ratio. However, partial SCFs might be perpendicularly oriented to the direction of heat flux in real cases, so the experimental results of thermal conductivity are less than the calculated ones. Fig. 11a to $11 \mathrm{c}$ also shows the calculated values of $\mathrm{k}$ with different $\left\langle\cos ^{2} \theta\right\rangle$ ranged from 0.33 to 0.6 . This clearly shows that the values of $\mathrm{k}$ will decrease with the increase of $\left\langle\cos ^{2} \theta\right\rangle$. The actual $\left\langle\cos ^{2} \theta\right\rangle$ of PDMS composites can be obtained by fitting the measured value into calculation. It was clear that the SCFNA method had a significant influence on the orientation of SCF. The component of the SCF along the heat direction will increase, and the component perpendicular to the heat direction will decrease after the application of external force field, so that the orientation of SCF will tend to be parallel to the heat flux with the increase of CR. Thus, $\left\langle\cos ^{2} \theta\right\rangle$ is gradually decreased with increasing CR (Fig. 11d to $11 \mathrm{f})$. When CR is 20,10 and 5 , the values of $\left\langle\cos ^{2} \theta\right\rangle$ are $0.52,0.53$ and 0.55 , respectively. The summary and comparison of calculated results and experimental data of the TCs of PDMS/SCF/GB composite samples with different CRs are presented in Fig. 11g and 11h.

In addition, as shown in Fig. 11d and 11e, the calculated TCs of PDMS/SCF/GB composites were increasingly higher than the experimental data after GB content exceeded the 
optimal value. The difference between calculated results and experimental data can be ascribed to the differences between ideal theoretical model and the real case. The TCN within the $\mathrm{PDMS} / \mathrm{SCF} / \mathrm{GB}$ composites was indestructible in ideal theoretical model. However, it can actually be destroyed in real cases when overmuch GBs were introduced into this composite system and led to a significant decrease of thermal conductivities.

According to the analysis above, the $\mathrm{TC}$ of $\mathrm{PDMS} / \mathrm{SCF} / \mathrm{GB}$ composite was closely related to the SCF orientation, thermal resistance of polymer in TCN, and the content of GBs. With the increase of $\mathrm{CR}$, the SCFs in $\mathrm{PDMS} / \mathrm{SCF} / \mathrm{GB}$ composite will trend to orient parallelly to the heat flow direction. Meanwhile, the distance reduction between two adjacent SCFs would bring significant thermal resistance decreasing for polymer layers in TCNs. The CR increasing and the addition of appropriate amount of GBs could be treated as normal processes of TCN conversion from high to low thermal dissipation, which ultimately led to the noticeable TC enhancement of the PDMS/SCF/GB composite.

\section{Conclusions}

A new theory of TCN conversion from high to low thermal dissipation was proposed in this study for TC enhancement of polymer composites. The PDMS/SCF/GB composite was selected to prove the correctness of this new theory from both theoretical and experimental perspectives. Two different approaches, including SCFNA method for the densification of TCNs and introducing GBs into composite system, were performed to realize the conductive network conversion in this composite system. The TC of $\mathrm{PDMS} / \mathrm{SCF}(30$ $\mathrm{wt} \%) / \mathrm{GB}(2 \mathrm{wt} \%)$ composites achieved the maximum value of $13.004 \mathrm{~W} / \mathrm{mK}$ after the realization of TCN conversion from high to low thermal dissipation. Compared with the samples fabricated using traditional compounding methods, this TC presented a one order of magnitude enhancement. In theoretical analysis, the ETC of PDMS/SCF/GB composite was calculated according to Nan model using the laws of equivalent $\mathrm{TC}$ and minimal thermal resistance. The high consistency between calculated results and experimental data verified the soundness of our theoretical analysis and assumptions. Our theory and method can provide researchers a new perspective for TC enhancement of polymer composites.

\section{Acknowledgement}

The authors thank the financial supports from National Natural Science Foundation of China (No. 52003019), Natural Science Foundation of Beijing Municipality (No. 2204090), Opening Project of State Key Laboratory of Molecular Engineering of Polymers (Fudan University) (No. k2021-14), and the Talents Introduction Project in Beijing University of Chemical Technology (No. buctrc201909).

\section{Conflict of Interest}

There is no conflict of interest.

\section{Supporting Information}

Not applicable

\section{References}

[1] L. Peng, Z. Xu, Z. Liu, Y. Guo, P. Li, C. Gao, Adv. Mater. 2017, 29, 1700589, doi: 10.1002/adma.201700589.

[2] W. Si, J. Sun, X. He, Y. Huang, J. Zhuang, J. Zhang, V. Murugadoss, J. Fan, D. Wu, Z. Guo, J. Mater. Chem. C, 2020, 8, 3463-3475, doi: 10.1039/C9TC06968B.

[3] S. Wang, Y. Liu, Y. Guo, Y. Lu, Y. Huang, H. Xu, D. Wu, J. Sun, Mater. Today Commun., 2020, 25, 101206, doi: 10.1016/j.mtcomm.2020.101206.

[4] Y. Du, Q. Zhang, Y. Huang, H. Xu, X. Bai, X. Zhang, H. Zhang, D. Wu, J. Sun, Compos. Commun., 2021, 24 100673, doi: 10.1016/j.coco.2021.100673.

[5] H. Zhang, X. Zhang, D. Li, X. Yang, D. Wu, J. Sun, Compos. Commun., 2020, 22, 100509, doi: 10.1016/j.coco.2020.1006509.

[6] W. Bauhofer, J.Z. Kovacs, Compos. Sci. Technol., 2009, 69, 1486-1498, doi: 10.1016/j.compscitech.2008.06018.

[7] H. Ku, H. Wang, N. Pattarachaiyakoop, M. Trada, Compos.

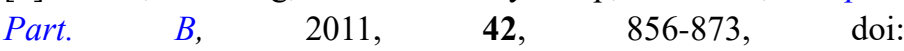
10.1016/j.compositesb.2011.01.010.

[8] M. Wang, X. Duan, Y. Xu, X. Duan, ACS Nano, 2016, 10, 7231-7247, doi: 101021/acsnano.6b03349.

[9] A.L. Moore, L. Shi, Mater. Today, 2014, 17, 163-174, doi: 10.1016/j.mattod.2014.04.003.

[10] Z. Ling, Z. Zhang, G. Shi, X. Fang, L. Wang, X. Gao, Y. Fang, T. Xu, S. Wang, X. Liu, Renew. Sustain. Energy Rev., 2014, 31, 427-438, doi: 10.1016/j.rser.2013.12.017.

[11] J. Sun, J. Shen, S. Chen, M. Cooper, H. Fu, D. Wu, Z. Yang, Polymers, 2018, 10, 505, doi: 10.3390/polym10050505.

[12] Y. Huang, S. Kormakov, X. He, X. Gao, X. Zheng, Y. Liu, J. Sun, D. Wu, Polymers, 2019, 11, 187, doi: 10.3390/polym11020187.

[13] J. Sun, Y. Zhao, Z. Yang, J. Shen, E. Cabrera, M.J. Lertola, W. Yang, D. Zhang, A. Benatar, J.M. Castro, D. Wu, L.J. Lee, Nanot, 2018, 29, 355304, doi: 10.1088/1361-6528/aacc59.

[14] L. Fan, J.M. Khodadadi, Renew. Sustain. Energy Rev., 2011, 15, 4-46, doi: 10.1016/j.rser.2010.08.007.

[15] H. Deng, L. Lin, M. Ji, S. Zhang, M. Yang, Q. Fu, Prog. Polym. Sci., 2014, 39, 627-655, doi: 10.1016/j.progpolymsci.2013.07.007.

[16] J. Gu, C. Liang, X. Zhao, B. Gan, H. Qiu, Y. Guo, X. Yang, Q. Zhang, D.-Y. Wang, Compos. Sci. Technol., 2017, 139, 83-89, doi: 10.1016/j.compscitech.2016.12.015.

[17] N. Burger, A. Laachachi, M. Ferriol, M. Lutz, V. Toniazzo, D. Ruch, Prog. Polym. Sci., 2016, 61, 1-28, doi: 10.1016/j.progpolymsci.2016.05.001.

[18] J. Wang, X. Zhang, Y. Liu, C. Xu, H. Zhang, D. Wu, T. Tan, X. Qin, J. Sun, L. Zhang, Compos. Sci. Technol., 2021, 202, 108582, doi: 10.1016/j.compscitech.2020.108582. 
[19] P. Zhang, Q. Li, Y. Xuan, Compos. Part A, 2014, 57, 1-7, doi: 10.1016/j.compositesa.2013.10.022.

[20] H.A. Babrekar, N.V. Kulkarni, J.P. Jog, V.L. Mathe, S.V. Bhoraskar, Mater. Sci. Eng. B, 2010, 168, 40-44, doi: 10.1016/j.mseb.2009.11.009.

[21] F. Zhao, D. Chen, S. Chang, H. Huang, K. Tong, C. Xiao, S. Chou, H. Zhong, Q. Pei, J. Mater. Chem. C, 2017, 5, 531-538, doi:10.1039/c6tc04934f.

[22] S. Hwang, E.I. Reyes, K.-s. Moon, R.C. Rumpf, N.S. Kim, JEMat, 2014, 44, 771-777, doi: 10.1007/s11664-014-3425-6.

[23] Y. Feng, C. He, Y. Wen, X. Zhou, X. Xie, Y. Ye, Y.-W. Mai, Compos. Sci. Technol., 2018, 160, 42-49, doi: 10.1016/j.compscitech.2018.03.009.

[24] T.M.L. Dang, C.-Y. Kim, Y. Zhang, J.-F. Yang, T. Masaki, D.-H. Yoon, Compos. Part B, 2017, 114, 237-246, doi: 10.1016/j.compositesb.2017.02.008.

[25] Y. Yao, X. Zhu, X. Zeng, R. Sun, J.-B. Xu, C.-P. ACS AMI, 2018, 10, 9669-9678, doi: 10.1021/acsami.8b00328.

[26] J. Hu, Y. Huang, Y. Yao, G. Pan, J. Sun, X. Zeng, R. Sun, J.-B. Xu, B. Song, C.-P. Wong, ACS AMI, 2017, 9, 1354413553, doi: 10.1021/acsami.7b02410.

[27] X. Zeng, J. Sun, Y. Yao, R. Sun, J.-B. Xu, C.-P. Wong, ACS Nano, 2017, 11, 5167-5178, doi: 10.1021/ascnano.7b02359.

[28] X. Cui, P. Ding, N. Zhuang, L. Shi, N. Song, S. Tang ACS AMI, 2015, 7, 19068-19075, doi: 10.1021/acsami.5b04444.

[29] W.-b. Zhang, Z.-x. Zhang, J.-h. Yang, T. Huang, N. Zhang, X.-t. Zheng, Y. Wang, Z.-w. Zhou, Carbon, 2015, 90, 242-254, doi: 10.1016/j.carbon.2015.04.040.

[30] J. Sun, J. Zhuang, J. Shi, S. Kormakov, Y. Liu, Z. Yang, D. Wu, J. Mater. Sci., 2019, 54, 8436-8449, doi: 10.1007/S10853019-03472-1.

[31] H. Wu, H. Xia, X. Zhang, H. Zhang, H. Liu, J. Sun, J. Nanophotonics, 2020, 14, 046003 doi: 10.1117/1.jnp.14.046003.

[32] C. Feng, H. Ni, J. Chen, W. Yang, ACS AMI, 2016, 8, 19732-19738, doi: 10.1021/acsami.6b03723.

[33] X. He, Y. Huang, Y. Liu, X. Zheng, S. Kormakov, J. Sun, J. Zhuang, X. Gao, D. Wu, J. Mater. Sci., 2018, 53, 14299-14310, doi: 10.1007/s10853-018-2618-4.

[34] F. Su, Z. Zhao, Y. Liu, W. Si, C. Leng, Y. Du, J. Sun, D. Wu, J. Polym. Eng., 2019, 39, 892-901, doi: 10.1515/polyeng2019-0086.

[35] X. He, Y. Huang, C. Wan, X. Zheng, S. Kormakov, X. Gao, J. Sun, X. Zheng, D. Wu, Compos. Sci. Technol., 2019, 172, 163-171. doi: 10.1016/j.compscitech.2019.01.009.

[36] S. Kormakov, D. Wu, J. Sun, X. Gao, X. He, X. Zheng, I. Skopincev, N. Memetov, A. Tkachev, Z. Zhi, Express Polym. Lett., 2019, 13, 713-723, doi: 10.3144/expresspolymlett.2019.60.

[37] H. Zhang, X. Zhang, Z. Fang, Y. Huang, H. Xu, Y. Liu, D. Wu, J. Zhuang, J. Sun, Recent J. Compos. Sci., 2020, 4, 180, doi: $10.3390 /$ jcs4040180.

[38] H. Chen, V.V. Ginzburg, J. Yang, Y. Yang, W. Liu, Y. Huang, L. Du, B. Chen, Prog. Polym. Sci., 2016, 59, 41-85, doi: 10.1016/j.progpolymsci.2016.03.001.
[39] C.-C. Teng, C.-C.M. Ma, K.-C. Chiou, T.-M. Lee, Compos. Part B, 2012, 43, 265-271, doi: 10.1016/j.compositesb.2011.05.027.

[40] D. Zhang, J. Sun, L.J. Lee, J.M. Castro, Eng. Sci., 2020, 10, 35-50, doi: 10.30919/es5e1002.

[41] X. Zeng, Y. Yao, Z. Gong, F. Wang, R. Sun, J. Xu, C.-P. Wong, Small, 2015, 11, 6205-6213, doi: 10.1002/smll.201502173.

[42] Z.S. Weng, K. Wu, F.B. Luo, F. Xiao, Q. Zhang, S. Wang, M.G. Lu, Compos. Part A, 2018, 110, 106-112, doi: 10.1016/j.compositesa.2018.04.016.

[43] Y. Yao, X. Zhu, X. Zeng, R. Sun, J.B. Xu, C.P. Wong, ACS AMI, 2018, 10, 9669-9678, doi: 10.1021/acsami.8b00328.

[44] S. Zhai, P. Zhang, Y. Xian, J. Zeng, B. Shi, IJHMT, 2018, 117 358-374, doi: 10.1016/j.ijheatmasstransfer.2017.09.067.

[45] M. Wang, N. Pan, Mater. Sci. Eng. R, 2008, 63, 1-30, doi: 10.1016/j.mser.2008.07.001.

[46] J. Sun, J. Zhuang, H. Jiang, Y. Huang, X. Zheng, Y. Liu, D. $\mathrm{Wu}$, Appl. Therm. Eng., 2017, 121, 492-500, doi: 10.1016/J.APPLTHERMALENG.2017.04.104.

[47] J. Zhuang, W. Hu, Y. Fan, J. Sun, X. He, H. Xu, Y. Huang, D. Wu, Microsyst. Technol., 2018, 25, 381-388, doi: 10.1007/s00542-018-3988-x.

[48] S. Kormakov, X. He, Y. Huang, Y. Liu, J. Sun, X. Zheng, I. Skopincev, X. Gao, D. Wu, PoCom, 2018, 40, 1819-1827, doi:10.1002/pc.24942.

[49] J. Zhuang, J. Sun, D. Wu, Y. Liu, R.R. Patil, D. Pan, Z. Guo, Adv. Compos. Hybrid Mater., 2021, 4, 27-35, doi: 10.1007/s42114-021-00204-5.

[50] Z. Han, A. Fina, Prog. Polym. Sci.,2011, 36, 914-944, doi: 10.1016/j.progpolymsci.2010.11.004.

[51] Y. Yao, J. Luo, X. Duan, T. Liu, Y. Zhang, B. Liu, M. Yu, Carbon, 2019, 141, 283-290, 10.1016/j.carbon.2018.09.043.

[52] A.G. Every, Y. Tzou, D.P.H. Hasselman, R. Raj, AcM\&M, 1992, 40, 123-129, doi:10.1016/0956-7151(92)90205-s.

[53] Y. Benveniste, JAP, 1987, 61, 2840-2843, doi: 10.1063/1.337877.

[54] D.A.G. AnP, 1935, 416, 636-664, doi: 10.1002/andp, 19354160705.

[55] C.-W. Nan, R. Birringer, D.R. Clarke, H. Gleiter, JAP, 1997, 81, 6692-6699, doi:10.1063/1.365209.

[56] E.T. Swartz, R.O. Pohl, RvMP, 1989, 61, 605-668, doi: 10.1103/RevModPhys.61.605.

[57] D. Wu, J. Sun, Y. Liu, Z. Yang, H. Xu, X. Zheng, P. Gou, Polym. Eng. Sci., 2017, 57, 268-274, doi:10.1002/pen.24408..

[58] J. Sun, D. Wu, Y. Liu, L. Dai, C. Jiang, Adv. Polym. Tech., 2018, 37, 1581-1591, doi: 10.1002/adv.21815.

[59] S. Jingyao, W. Daming, L. Ying, Y. Zhenzhou, G. Pengsheng, Polym. Eng. Sci., 2018, 56, 952-960, doi: 10.1002/pen.24651.

[60] J. Zhuang, D.M. Wu, H. Xu, Y. Huang, Y. Liu, J.Y. Sun, Int. Polym. Proc., 2019, 34, 231-238, doi: 10.3139/217.3726. 


\section{Author information}

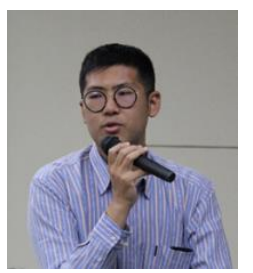

Jingyao Sun is an associate professor in the College of Mechanical and Electrical Engineering at Beijing University of Chemical Technology. Dr Sun's research interests focus on advanced polymer manufacturing and processing technology and equipment for polymer-based functional composites.

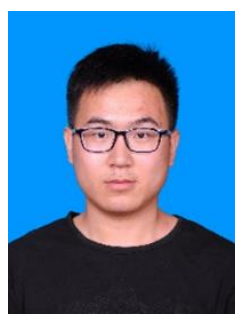

Xiaowen Zhang is a postgraduate student in the College of Mechanical and Electrical Engineering, Beijing University of Chemical Technology. His main research interests include advanced manufacturing and application of polymer nanocomposites, polymer injection molding and extrusion.

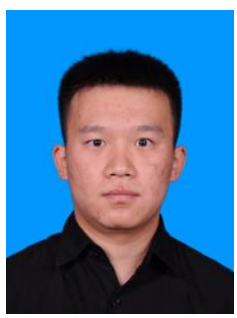

Qingyuan Du is a postgraduate student in the College of Mechanical and Electrical Engineering, Beijing University of Chemical Technology. The main research direction is the advanced manufacturing and application of polymer nanocomposites, $3 D$ printing preparation of polymer structure and function integrated composite materials processing methods.

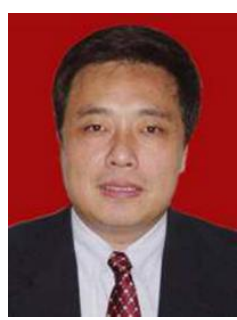

Daming Wu is a professor in the College of Mechanical and Electrical Engineering at Beijing University of Chemical Technology. In 1992, he graduated from Moscow Institute of Chemical Machinery, Russia. His research interests include plastic machinery, high performance polymer composites, polymer micro/nano manufacturing, polymer precision molding, etc. At present, he is the deputy director of Beijing Key Laboratory of Preparation and Processing of New Polymer Materials, an expert of the Expert Committee of China Plastics Processing Association, an expert of the Expert Committee of China Plastics Machinery Industry Association, and a standing director of Beijing Plastics Processing Association.

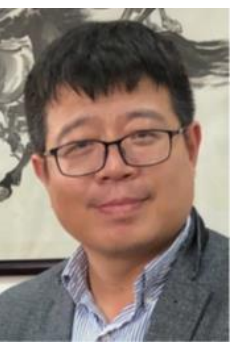

Zhanhu Guo earned his Ph.D. degree in chemical engineering from Louisiana State University in 2005. He received his threeyear postdoctoral training in mechanical and aerospace engineering at the University of California Los An-geles. He is a Professor in Chemical and Biomolecular Engineering directs the Integrated Composites Laboratory at The University of Tennessee, Knoxville. His current research interests are in the areas of optoelec-tronic, electric, and magnetic materials for solar cells, catal-ysis, sensing, and electronics.

Publisher's Note Engineered Science Publisher remains neutral with regard to jurisdictional claims in published maps and institutional affiliations. 